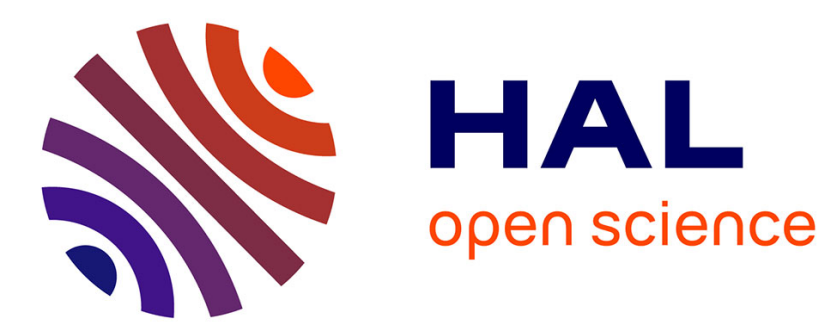

\title{
ENERGY-POOLING PROCESSES IN \\ LASER-EXCITED ALKALI VAPORS : AN UPDATE ON EXPERIMENTS
}

\author{
M. Allegrini, C. Gabbanini, L. Moi
}

\section{- To cite this version:}

M. Allegrini, C. Gabbanini, L. Moi. ENERGY-POOLING PROCESSES IN LASER-EXCITED ALKALI VAPORS : AN UPDATE ON EXPERIMENTS. Journal de Physique Colloques, 1985, 46 (C1), pp.C1-61-C1-73. 10.1051/jphyscol:1985106 . jpa-00224475

\section{HAL Id: jpa-00224475 https://hal.science/jpa-00224475}

Submitted on 1 Jan 1985

HAL is a multi-disciplinary open access archive for the deposit and dissemination of scientific research documents, whether they are published or not. The documents may come from teaching and research institutions in France or abroad, or from public or private research centers.
L'archive ouverte pluridisciplinaire HAL, est destinée au dépôt et à la diffusion de documents scientifiques de niveau recherche, publiés ou non, émanant des établissements d'enseignement et de recherche français ou étrangers, des laboratoires publics ou privés. 


\title{
ENERGY-POOLING PROCESSES IN LASER-EXCITED ALKALI VAPORS : AN UPDATE ON EXPERIMENTS
}

\author{
M. Allegrini, C. Gabbanini and L. Moi \\ Istituto di Fisica Atomica e Molecolare del C.N.R., \\ Via del Giardino, 7, Pisa, Italy
}

\begin{abstract}
Résumé - Les processus d "energy pooling"dans les vapeurs atomiques alcalines excıtées par laser sont passês en revue. On présente en particulier les résul.tats les plus récents obtenus pour le sodium.
\end{abstract}

\begin{abstract}
We present a short review of energy pooling experiments in laser excited alkali vapors, for low atom density and low radiation intensity. Special emphasis is devoted to the most recent results obtained in sodium vapor.
\end{abstract}

\section{I - INTRODUCTION}

Laser excitation of dense vapors produces a large variety of atomic and molecular processes /1/, that are not easily separated in the experiments. Thus these kinds of experiments offer on the one hand a very rich and interesting way of studying atom-atom and atom-e.m. fjeld interactions, but on the other hand they suffer of the difficulties associated with the correct interpretation of the observed phenomena. To simplifly this matter it is convenient to select in the experiment the proper values of the parameters that may determine the dominant effect, namely the laser power density, the atom and molecule densities in the vapor and, last but not least, the temporal characteristics of the laser /2/. By increasing the laser power density $I_{L}$ an enhancement of the multiphoton ionization is obtained, while increasing the atom density $N$ collisional processes in the vapor are amplified. It is not hard to image that by changing both $I_{L}$ and $N$ very complicated and interesting situations may be created; moreover this picture is further complicated by any molecules that are present in the vapor $/ 3 /$.

Here we consider the experiments performed at low laser power density (1-10Wcm ${ }^{-2}$ ) and low atom density $\left(10^{11}-10^{13} \mathrm{~cm}^{-3}\right)$ while other processes are extensively treated elsewhere in this book. The first requirement assures that the multiphoton or processes induced by the laser field are negligible, the second that the secondary collisional processes, such as the electron superelastic collisions, are negligible. More precisely we consider the experiments where the dominant effects are the energy pooling collisions, i.e. the binary inelastic collisions of the type

$$
A^{*}(n p)+A^{*}(n p) \rightarrow A_{2}^{* *}(n 1)+A(n s)
$$


where $A(n s)$ is the alkali atom in the ground state, $A^{*}(n p)$ is the laser excited state, $A^{* *}(n I)$ is any excited state above the first and $A_{2}^{+}$is the molecular ion. These collisions require, in the limit of a few $\mathrm{kT}$, near resonance between the initial and final state energies.

These processes have been observed for the first time in sodium $/ 4 /$ at $10^{12}-10^{13} \mathrm{~cm}$ and very low laser intensity $\left(\leqslant 10 \mathrm{Wcm}^{-2}\right)$; later on they have been recognized /5-7/ as the primary mechanism for producing both the seed electrons and the highly-excited states involved in the complete ionization experiments. The first example of this kind of experiment was succesful again in sodium /8/. Thus the energy pooling collisions deserve deep investigations not only for the understanding of the long range interactions in atoms, but also for the achievement of ionization with very moderate laser powers.

There are essentially three ways to detect the effects produced by a resonant laser excitation of a vapor: look at the fluorescence spectrum (i.e. detect the photons), detect the ions, either by collection of the total ion yield or by mass-resolved ion spectra, and collect the electrons. The detection apparatus can be considerably different in the three cases and also the geometry of the experiment has a fundamental influence on the results. The combination of these different techniques has given a substantial agreement on the phenomena investigated and we have now a qualitative picture of the general problem plus reliable quantitative results. In the first observation of process (1a) / $/$ the apparatus was very simple and only the fluorescence spectrum was reported; in addition to the radiation from highly excited states observed in ref./4/, Leventhal and coworkers /9,10/ were the first to report, from the same experiment, also ion formation, both $\mathrm{Na}^{+}$and $\mathrm{Na}_{2}^{+}$produced by process (1b). The experimental conditions of ref./4/ and $/ 9,10 /$ are basically the same, i.e. low atom density $\leqslant 10^{13} \mathrm{~cm}^{-3}$ and low laser power density $\lesssim 10 \mathrm{wcm}^{-2}$; the two experiments proved that the excited states and the ions are produced by the same entrance channel, the collisions $3 p+3 p$, which gives the energy transfer (1a) in the first case and the associative ionization (1b) in the second case. Ion analysis, with various tecniques for extraction and collection, have been used in sodium also by Carré et al. /11/. Merit of work /11/ is to have defined the values of laser power for which the laser field, although not strong enough to produce directly multiphoton ionization, may assist the simple associative ionization (1b) and the values of atom density for which the electron superelastic collisions become important. These results are also in good agreement with those obtained by weiner and coworkers on beam experiments /12/. A definite proof of process (1) as the primary mechanism producing the low energy seed electrons has been given in ref./7/. This experiment is very important-because it introduces, for the first time, electron spectrometry to resolve the energy spectrum of the electrons created in the vapor.

It is worth mentioning here that in the alkalis, $A^{*}(n p)+A^{*}(n p)$ connects to many electronic states $A_{2}^{* *}$ of the neutral molecule. The interesting side of this process is that the molecular states $A_{2}^{* *}$ have not necessarily the simmetry of a state allowed by dipole selection rules for one-photon absorption from the ground state of the dimer. Thus this collisional spectroscopy may be promising for producing triplet excited states of the alkali molecules /13/ and studying their possible application for new tunable laser sources. 
II - RECENT EXPERIMENTS AND RESULTS

If the comprehension of the phenomena occurring in a dense vapor excited by resonant laser radiation is not straightforward because of their complexity, much more care has to be taken for a quantitative analysis. The first step is to perform the experiment with specific $I_{L}$ and $N$ to single out the process of interest; the energy pooling process (1) is usually made the dominant effect by reducing $N$ and $I_{L}$. Then the main problem to proceed from the early qualitative observations to quantitative reliable measurements of the rate coefficients $K$ or cross sections $\sigma$ for processes (1a) and (1b), is the determination of the excited atom density $\mathrm{N}_{\mathrm{np}}$. An indetermination in excited atom density has indeed severe consequences on the values of $\mathrm{K}$ and $\sigma$ because of the square dependence of the energy pooling processes from $\mathrm{N}$, as can be easily shown by writing the rate equations. In the experiments where all the processes other than the energy pooling can be neglected the following simplified rate equation applies:

$\dot{\mathrm{N}}_{\mathrm{nl}}=\mathrm{K}_{\mathrm{nl}} \mathrm{N}_{\mathrm{np}}^{2}-\mathrm{N}_{\mathrm{nl}} \sum_{\mathrm{n}^{\prime} l^{\prime}} \mathrm{A}\left(\mathrm{nl}, \mathrm{n}^{\prime} \mathrm{l}^{\prime}\right)$

where $\mathrm{N}_{\mathrm{n}}$ is the population of the $\left.n\right]$ level, $\mathrm{K}_{\mathrm{n}}$ is the rate coefficient for process $(1 a)$ and $A\left(n l, n^{\prime} l^{\prime}\right)$ is the spontaneous transition probability for $\left.|n l>\rightarrow| n^{\prime} l^{\prime}\right\rangle$. Analogous equation can be written for process (1b) when the associative ionization rate coefficient $K_{A I}$ has to be determined. Solution of eq.(2) at the steady-state gives

$K_{n I}=<\sigma_{n I} v>=\left(N_{n I} / N_{n p}^{2}\right) \quad \sum_{n^{\top}}, A\left(n I, n^{\prime} I^{\prime}\right)$

where $v=\sqrt{8 \mathrm{kT} / \pi \mu}$ is the relative mean interatomic velocity. The quantity measured in the experiments is the intensity of the fluorescence lines or, more often, the intensity ratio of the emission from the highly excited state nl. to the laser excited state np and from np to the ground state ns. The intensity per unit solid angle, $I_{i k}$, of a fluorescence line from level $i$ to level $k$, as detected by the apparatus, is related to the population of level i through the relation

$I_{i k}=\hbar \omega_{i k} A_{i k}^{N}{ }_{i} \varepsilon_{i k} V / 4 \pi$

where $\omega_{i k}$ is the transition frequency, $\varepsilon_{i k}$ is a factor which takes into account the instrumental response of the detecting apparatus and $V$ is the fluorescence volume. In .case of self trapping the natural radiative decay rate expressed by the spontaneous transition probability $A_{i k}$ has to be changed with an effective rate which defines a trapped lifetime $\tau^{3 *}$ compared to the natural Iifetime $\tau_{0}$. Therefore the experiment involves also the determination of the effective laser-vapor interaction volume and the correct treatment of the radiation trapping phenomenon. Because of the self trapping, the apparent lifetine of the excited atom population increases, the diffusion of the excited atoms makes the interaction volume greater than the laser beam cylinder and also the radiation from the upper levels to the first $p$-level is attenuated with a change in the branching ratios of the higher nl-levels.

These difficulties have been overcome with various experimental approaches, that are briefly reviewed in the following while the results will be summarized in Table 1. Chéret et al. /14/, in their study of the Penning and associative ionization of $5 p$ rubidium atoms with highly lying levels $(6 \mathrm{~d}, 8 \mathrm{~s}, 7 \mathrm{~d})$, used the photoinization process 
$\mathrm{Rb}(\mathrm{nl})+\mathrm{h} \nu \rightarrow \mathrm{Rb}^{+}+\mathrm{e}^{-}$

obtained with a second laser, to determine the concentration of the excited atoms. The same method was applied /15/ for the investigation of the energy pooling process

$R b(5 p)+R b(5 p) \rightarrow R b(5 d)+R b(5 s)$

A $\mathrm{cw}$ dye laser $(\boldsymbol{\Delta} \nu \approx 25 \mathrm{GHz}$ and maximum power $250 \mathrm{~mW})$ excites the rubidium atoms to the $5 \mathrm{p}_{3 / 2}$ level at a vapor density in the range $5 \times 10^{11}-10^{13} \mathrm{~cm}^{-3}$; thus the experimental conditions are similar to those of the previous experiments in sodium $14,9,10 /$. The population density of both the $5 p$ and $5 d$ levels is deduced from the measurement of the $\mathrm{Rb}^{+}$ion current produced through process (5) with a second cw $\mathrm{Ar}^{+}$laser that crosses the dye laser at right angles. By shifting the $\mathrm{Ar}^{+}$laser beam in a vertical plane also the spatial distribution of the excited states can be followed. The interaction volume is estimated by the overlapping of the two laser beams and absolute calibration of the ion current is made on a polarized plate inside the cell. The uncertainty on the rate coefficient for process (6) measured with this method (and reported in table 1 ) depends upon the accuracy of the photoionization cross-sections. Direct photoionization of the excited atoms has been used also in a beam experiment on sodium /16/ to measure the 3p atom density necessary for the determination of the associative ionization cross section for the process

$\mathrm{Na}(3 p)+\mathrm{Na}(3 p) \rightarrow \mathrm{Na}_{2}^{+}+\mathrm{e}^{-}$

The basic idea of this work is to get $\mathrm{N}_{3 \mathrm{p}}$ from the process

$\mathrm{Na}(3 \mathrm{p})+\mathrm{h} v \rightarrow \mathrm{Na}^{+}+e^{-}$

which gives a signal proportional to the $3 p$ density, and to reduce the $\mathrm{Na}^{+}$ production measurement to a ratio measurement of $\mathrm{Na}_{2}^{+}$and $\mathrm{Na}^{+}$densities. Measurement of an intensity ratio avoids the difficult determination of the absolute efficiency of the detecting apparatus, however, as we have already mentioned for the case of fluorescence detection, great care has to be taken to define the volume over which the signals are collected. In this particular experiment, for example, the volume for the $\mathrm{Na}^{+}$ions is simply defined by the laser beam that photoionizes the $3 p$ atoms whereas the $\mathrm{Na}^{+}$ions, produced in process (7), are found in an enlarged effective volume created by the diffusion of the sodium atoms trapped in the $3 \mathrm{p}$ level. Another original way /17/ to measure excited state density in non-equilibrium, non steady-state situations is based on the increase of the trapped lifetime as a function of the total atom density. Leventhal and coworkers are now using this technique for $\mathrm{Na}(3 \mathrm{p})$ determination in their experiments. Problems with early measurements (cfr. table 1) are probably related to the determination of the interaction volume in a cell that is not uniformely heated and not closed because of the holes for ion extraction. 
This brief overview does not exhaust all the solutions devised in the different experiments, however we will consider only two more approaches, used for the specific measurement of the energy transfer cross section for the $5 \mathrm{~s}$ and $4 \mathrm{~d}$ levels of sodium, which are more relevant to the work reported in the next paragraph. The two methods are based on opposite basic idea: the first /18/ takes advantage of the self trapping, to measure the spatial and temporal distribution of the excited atoms, the second /19/ makes an absolute calibration of the apparatus at Low temperature when the self trapping is avoided. Huennekens and Gallagher /18/ have used pulsed excitation, tuned to the $\mathrm{D}_{2}$ resonance line to produce the $\mathrm{Na}(3 \mathrm{p}$ ) atoms $\left(I_{L} \cong 60 \mu \mathrm{J}\right.$ in pulses of $\left.\sim 5 \mathrm{nsec}, \Delta \lambda \cong 0.5 \mathrm{~cm}^{-1}\right)$; the laser beam diameter is chosen in order to match the fundamental mode decay rate as a function of the time of the excited atom spatial distribution in presence of self trapping. A second laser, operating in $\mathrm{cw}$ single mode at very low power $(\sim 100 \mu \mathrm{W})$ and highly detuned from the $\mathrm{D}_{2}$ line center $(\Delta y>2.4 \mathrm{GHz})$ to avoid optical pumping distorsion, crosses at right angles the pulsed laser beam. The measurement of the change in transmission of the $\mathrm{cw}$ laser, following the pulsed excitation, is used to obtain the fraction of atoms in the $3 p$ state as a function of time. The spatial distribution of $3 p$ atoms is obtained by measuring the resonance fluorescence intensity and using the Holstein-vanTrigt theory $/ 20 /$ for the diffusion across an infinite slab of trapped atoms. This theory applies correctly to this experiment because the geometry of the collision cell has been properly studied and the optical depths are high. Once the $3 p$ atom density is known the rate constants for

$\mathrm{Na}(3 \mathrm{p})+\mathrm{Na}(3 \mathrm{p}) \rightarrow \mathrm{Na}(5 \mathrm{~s}, 4 \mathrm{~d})+\mathrm{Na}(3 \mathrm{~s})$

are simply obtained by solving the rate equations of the process and by measuring the ratios of the transitions $5 s-3 p, 4 d-3 p$ and $3 p-3 s$. The same technique for $N_{3 p}$ density determination has been employed /37/ also for the measurement of the associative ionization cross-section (process (7)). As noted before the volume integral of $\mathrm{N}_{3 p}$ and the $\mathrm{N}_{3 p}$ density distribution is needed. Huennekens and Gallagher in this work have used two other independent methods for $N$ determination, one based on the total 3p-3s fluorescence intensity and the second $3 p$ on the ratio between $5 s-3 p$ and $3 p-3 s$ fluorescence intensity. The three techniques give results which agree within their uncertainties. In the experiment of ref./19/ the interaction volume is accurately determined in a capillary cell entirely illuminated by the laser beam (a broadband cw dye laser of low power $\Delta$ W $^{-2}$, tuned to the $D_{2}$ resonance line); the volume is also reduced to $4 \times 10^{-4} \mathrm{~cm}^{-3}$ by a trasverse narrow slit $(\cong 150 \mu \mathrm{m})$. The $\mathrm{Na}(3 \mathrm{p})$ atom density is determined first at . low temperature $\mathrm{T}$ through the saturation of the resonance fluorescence and then at the temperature $\mathrm{T}^{\prime}$, high enough to allow observation of process (9), simply by the relation

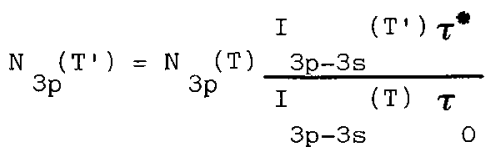

The trapped lifetime $\tau^{*}$ of the $3 p$ level is correctly given in this experiment by the Milne theory /21/ which, contrary to the Holstein-vanTrigt treatment, is valid for thin optical depths. Having the $\mathrm{N}_{3 p}$ value, the rate constants for process (9) are determined, as in the approach of Huennekens and Gallagher, by measuring fluorescence intensity ratios. The results of these two experiments have settle the previously lange discrepancies in sodium and are closer to the values 
of energy pooling cross sections for other alkalis.

Special attention deserves the experiment of Le Gouët et al. /7/. Although the relevant aim of this work is the direct observation of the superelastic collisions, that are outside our subject, it also shows unambiguously the role of the energy pooling processes in the production of the seed electrons. Sodium atoms in a beam of density $10^{13} \mathrm{~cm}^{-3}$ are excited to the $3 \mathrm{p}_{3 / 2}$ level by a cw dye laser of low intensity $\left(\approx 3 \mathrm{wcm}^{-3}\right)$. The multiphoton or laser assisted processes are then completely negligible as in the experiment of ref. $/ 4,9,10 /$. The detection apparatus consists of a cylindrical mirror analyzer that resolves the energy spectrum of the electrons emerging from the interaction zone. These spectra contain a number of peaks corresponding to the low-energy electrons produced by the associative ionization and to electrons created from the various excited states by the ionizing collisions

$\mathrm{Na}(3 p)+\mathrm{Na}(\mathrm{nl}) \rightarrow \mathrm{Na}(3 \mathrm{~s})+\mathrm{N}^{+}+e^{-}$

In particular peaks at energy values of electrons coming from atoms in the $5 s$ and $4 d$ states have been identified and an indirect estimation of the energy transfer cross-sections $\sigma_{5 \mathrm{~s}}$ and $\sigma_{4 \mathrm{~d}}$ has been made. The low energy electrons are then heated by one or more superelastic collisions leading to further ionization. However the interesting point from our point of view is that, in the conditions of low power laser intensity and relatively low atom density, the dominant processes are purely collisional.

The energy pooling processes have not received so far much attention from a theoretical point of view; for sodium, to which so many experiments have been devoted, there is only the calculation by Kowalczyk /22/ who considered the single procéss

$\mathrm{Na}(3 p)+\mathrm{Na}(3 p) \rightarrow \mathrm{Na}(4 \mathrm{~d})+\mathrm{Na}(3 \mathrm{~s})$

He uses a model first introduced by Borodin and Komarov/23/ for the calculation of the cross section for collision between two $6 \mathrm{p}$ caesium excited atoms. This model considers the adiabatic terms for the quasi molecule $\mathrm{Na}$ corresponding to the configurations $3 p+3 p$ and $3 s+4 d$ and it is valid only for large interatomic distances $(\mathrm{R}>20 \mathrm{a} \cdot \mathrm{u}$.$) because of the asymptotic approximations made. In the$ interaction Hamiltonian only the terms important at large distance, namely the exchange and dipole-dipole interactions, are taken into account. The contribution of ion-pair configurations is also neglected and the eigenfunctions are not orthogonalized. The error so introduced is minor for $R>35 a$. $u$. but it is important at shorter distances. Neverthiess the order of magnitude of the cross section calculated by Kowalczyk matches the experimental results, as shown in table 1. The same method has been applied by Barbier and Chéret $/ 15 /$ for the calculation of the cross section relative to process (6). This is a more favourable case than the one studied by Kowalczyk because the energy defect $\left(\Delta E \cong 68 \mathrm{~cm}^{-1}\right.$ ) is small and the main contributions come in the validity range of the model. Barbier and Chéret consider also the ionic $\left(\mathrm{R}^{+}+\mathrm{R}^{-}\right)$crossing curves for both the configurations $5 p+5 p$ and $5 d+5 s$; however this contribution is negligible in the $\mathrm{Rb}(5 \mathrm{p})+\mathrm{Rb}(5 \mathrm{p})$ reaction. The agreement between experimental and theoretical values is certainly better (cfr. table 1) for rubidium than sodium. However ab initio calculations of the potential energy curves for diabatic molecular states connecting to $3 s+n 1$ ( $w i$ th $n]=3 s, 4 s, 5 s, 3 p, 4 p, 3 d, 4 d$ and $4 f$ ) and 
$3 p+3 p$ are in progress for sodium /24/. These calculations are considered "complete" because both the Multi-Configuration-Self-Consistent-Field energy and the remaining energy of electronic correlation are calculated. The MC-SCF energy is obtained by an integrated density functional; then the coupling matrix elements for the various states are constructed and reliable quantitative determinations for all the energy pooling collisions from $3 p+3 p$ to the levels mentioned above are to be expected.

\section{III - AN UPDATE EXPERIMENT IN SODIUM}

In sodium, beside the $5 \mathrm{~s}$ and $4 \mathrm{~d}$, there is the level $4 \mathrm{f}$ which is close in energy to $3 p+3 p$; as an immediate extension of our early work, we have now measured the cross section for the process

$\mathrm{Na}(3 p)+\mathrm{Na}(3 p) \rightarrow \mathrm{Na}(4 f)+\mathrm{Na}(3 \mathrm{~s})$

This measurement was not done before mainly because the emission of the $4 f$ state is in the near infrared $(1.84 \mu \mathrm{m})$, a region far beyond the sensitivity of the optical apparatus used in previous experiments. However process (13) is of particular interest because the $4 f$ state lies very close to the $4 d\left(\Delta \mathrm{E} \simeq 38 \mathrm{~cm}^{-1}\right)$, therefore we get also informations on the process

$\mathrm{Na}(3 \mathrm{~s})+\mathrm{Na}(4 \mathrm{~d}) \frac{\mathrm{K}}{\mathrm{K}^{\prime}} \mathrm{Na}(3 \mathrm{~s})+\mathrm{Na}(4 f)$

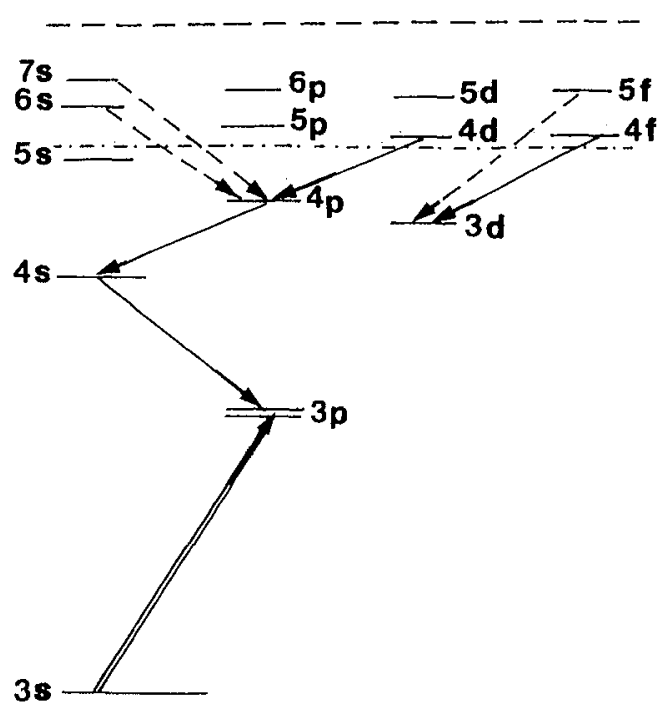

Fig. 1 - Simplified energy level scheme of $\mathrm{Na}$. The dashed line corresponds to the ionization limit and the dashed-dotted line to twice the energy of the $3 p$ level. The arrows correspond to the transitions observed in this experiment; the transitions indicated with a dashed line are present only in the cell. with buffer gas. 
Our apparatus has been sligthly modified to include a monochromator with a grating for the infrared and a PbS detector for the near infrared. The laser used to excite the sodium atoms to the $3 p_{3 / 2}$ level is a single mode dye laser of maximum power $120 \mathrm{~mW}$ and actively stabilized to a $\Delta \boldsymbol{\nu} \cong 1 \mathrm{MHz}$, with a frequency drift of less than $\cong 100 \mathrm{MHz} /$ hour. A single mode dye laser is not necessary in principle, however its stability characteristics have reduced the fluctuations of the laser intensity. To improve the signal-to-noise ratio the laser beam has been modulated and phase sensitive detection has been used. Further a second chopper has been used to switch on/off the beam at very low frequency $(\sim 0.4 \mathrm{~Hz})$; the same function generator which drives the chopper also triggers a multichannel analyzer system used for signal averaging. With $\mathrm{N}=2^{6}$ cycles recorded on the multichannel analyzer the statistical error decreases of a factor $1 / \sqrt{\mathrm{N}}=1 / 2^{3}$. At the end of each cycle the signal was displayed on the analyzer and chart recorded. Since each recording on the multichannel analyzer lasted $5 \mathrm{sec}$ the single mode dye laser has been very useful to keep the excitation stable on the maximum of the $\mathrm{D}_{2}$ line. As usual in our experiments the quantity measured is the intensity of the fluorescence lines. In figure 1 a scheme of the energy levels involved are reported with the observed transitions. We have used two cells, one with pure sodium and a second containing sodium plus a few torr of a buffer gas. The temperature has been kept to the lowest values allowing detection of the fluorescence from the desired levels without having fluorescence from higher levels which assures that the secondary collisional or ionizing processes are avoided. The cross section $\sigma_{4 f}$ for process (13) has been determined relatively to $\sigma_{4 d}$, that was measured in an "absolute" way, as mentioned before /19/. This approach is reasonable because the volume from which we observe the fluorescence of the $4 \mathrm{~d}$ and $4 \mathrm{f}$ levels is the same, the laser intensity is stable and no radiation trapping is present for these high levels. Moreover the emission $4 \mathrm{~d}-4 \mathrm{p}$ is in the same near infrared spectraI range of the $4 f-3 d$ emission so that we can measure their intensities with the same detecting apparatus and obtain directly the population ratio of the $4 \mathrm{~d}$ and $4 f$ levels. The relation between $\mathrm{N}_{4 f}$ and $\mathrm{N}_{4 d}$, necessary for the determination of $\mathrm{K}_{4 f}$ and $K_{4 d}$, has been obtained by solving the rate equations in steady-state conditions for both levels. The complete treatment includes processes such as radiative decay from higher levels, electron impact ionization, photoionization and Penning ionization in collisions with $3 p$ atoms and the solution is rather complicated. However in our experiment we have worked with atom density and laser power density low enough to make these terms negligible; we have instead taken into account the exchange term $4 f-4 d$. The equations are therefore

$\dot{N}_{4 d}=(A(4 d, 3 p)+A(4 d, 4 p)) N_{4 d}+K_{4 d} N_{3 p}^{2}-K N N_{4 d}+K^{\prime} N_{4 f}$

$\dot{N}_{4 f}=A(4 f, 3 d) N_{4 f}+K_{4 f} N_{3 p}^{2}+K^{2} N_{4 d}-K^{\prime} N N_{4 f}$

where. $K$ and $K$ ' are the rate coefficients of process (14); they are related to each other /25/ through the level degeneracy $\mathrm{K} / \mathrm{K}^{\prime}=\left(\mathrm{g}_{4 f^{\prime}} / \mathrm{g}_{4 \mathrm{~d}}\right) \exp (-\Delta E / k T)=1.26$. The solution of eq.(15) and (16) at the steady-state yields for the population ratio

$$
\frac{N_{4 f}}{N_{4 d}}=\frac{(A(4 d, 3 p)+A(4 d, 4 p)+K N)^{2} K_{4 f}+\langle A(4 d, 3 p)+A(4 d, 4 p)+K N) K K}{4 d^{N}} \frac{\left((A(4 d, 3 p)+A(4 d, 4 p)+K N)\left(A(4 f, 3 d)+K^{\prime} N\right)-K K^{\prime} N^{2}\right) K_{4 d^{2}}+((A,(4 d, 3 p)+A(4 d, 4 p)+K N) K}{\left.4 f^{+K K} 4 d^{N}\right) K^{\prime} \cdot N}
$$


The value $\mathrm{N}_{4 f^{\prime}} / \mathrm{N}$, for a fixed laser intensity and a given temperature, i.e. for a specific atom density $\mathrm{N}$, is obtained by measuring the intensity ratio

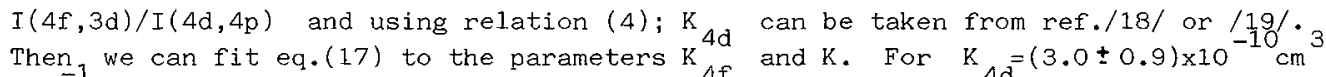
$\sec ^{-1}$ the best fit has given the values

$\mathrm{K}_{4 \mathrm{f}}=(5.6 \pm 2.2) \times 10^{-11} \mathrm{~cm}^{3} \mathrm{sec}^{-1} \quad ; \mathrm{K}=(9.8 \pm 4.9) \times 10^{-9} \mathrm{~cm}^{3} \mathrm{sec}^{-1}$.

In figure 2 the data for $\mathrm{N}_{4 f} / \mathrm{N}_{4 d}$, with the best fit curve, are reported as a function of the temperature.

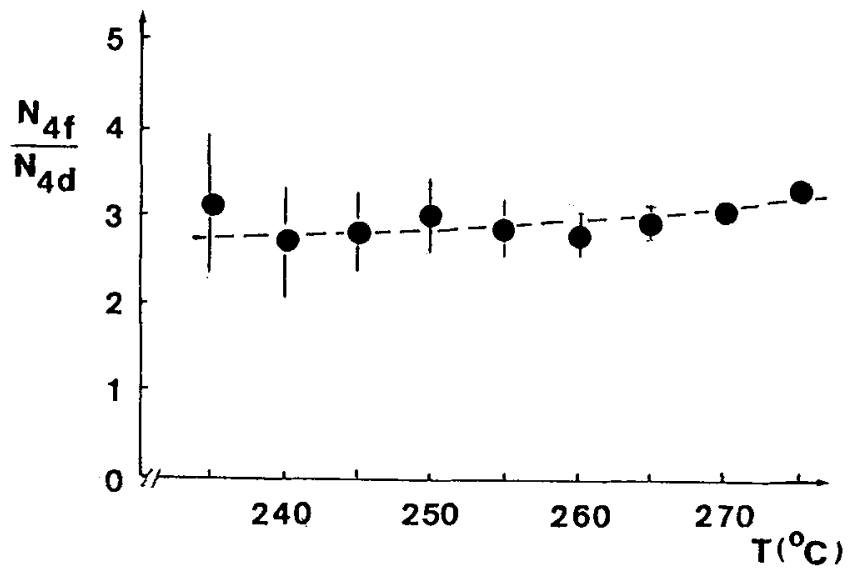

Fig. $2-\mathrm{N}_{4 \mathrm{f}} / \mathrm{N}_{4 \mathrm{~d}}$ ratio versus temperature. The dots represent the data and the dashed curve the best fit to eq. (17).

The indetermination on $\mathrm{K}$ shows, as expected, that this experiment is not the best way to investigate process (14); a simple way to perform this experiment would be to excite by two photon the transition 3s-4d and then detect the sensitized fluorescence $4 f-3 d$. In this context, however, it is more interesting to see the variation of $K$ when the exchange collisions $4 f-4 d$ are neglected. In this case the solution of the simplified rate equations yields

$K_{4 f} / K_{4 d}=\left(N_{4 f} / N_{4 d}\right)((A(4 f, 3 d) /(A(4 d, 3 p)+A(4 d, 4 p)))$

which gives the value $\mathrm{K}_{4 \mathrm{f}}=(6.0 \pm 2.4) \times 10^{-11} \mathrm{~cm}^{3} \mathrm{sec}^{-1}$. It is evident that the effect of process (14) on $K_{4 f}$ is not very strong at the temperatures of our experiments; since $K_{4 d}$ is about one order of magnitude bigger than $K_{4 f}$ this effect is even less important and this fully justifies why in both the experiments of ref./18/ and /19/ was neglected. At higher temperatures, however, the exchange $4 d-4 f$ increases and according to what observed by Huennekens and Gallagher and to our results shown in figure 2 seems to depopulate the 4 d level. The theoretical. work which is in progress /24/ is expected to be accurate enough to explain also the different magnitude of the energy pooling cross section for $4 d$ and $4 f$ 
$\left(\sigma_{4 \mathrm{~d}}=(3.2 \pm 1.1) \times 10^{-15} \mathrm{~cm}^{2}\right.$ compared to $\sigma_{4 \mathrm{f}}=(5.7 \pm 2.3) \times 10^{-16} \mathrm{~cm}^{2}$ at $\left.\mathrm{T}=250^{\circ} \mathrm{C}\right)$; as a first comment we may say that the selection rules apparently favour the collisions where the total atomic angular momentum is unchanged. Our combined results for $\sigma_{5 \mathrm{~s}}, \sigma_{4 \mathrm{~d}}$ and $\sigma_{4 f}$ indicates that the production of $3 d$ atoms (fluorescence from the $3 \mathrm{~d}$. level was observed in ref./4/ as one of the most intense) is through cascade from $4 d$ and $5 s$ via the $5 p$ level and direct radiative decay from the $4 f$ level, which is in contrast with the results of Krasinski et al. $/ 26 /$.

Similar investigations with a cell containing a buffer gas have given signals from higher levels, such as the 5f. Power dependence (see for example figure 3 regarding the $5 f$ level) indicates that these levels are primarily populated not by energy transfer collisions

$\mathrm{Na}(3 \mathrm{p})+\mathrm{Na}(3 \mathrm{p}) \rightarrow \mathrm{Na}(5 f)+\mathrm{Na}(3 \mathrm{~s})$

but rather through recombination. Neverthless, since fluorescence from the $5 f$ level was not observed in the cell without buffer gas, we can give an upper limit to the rate coefficient of process (19): $k<10^{-13} \mathrm{~cm}^{3} \mathrm{sec}^{-1}$.

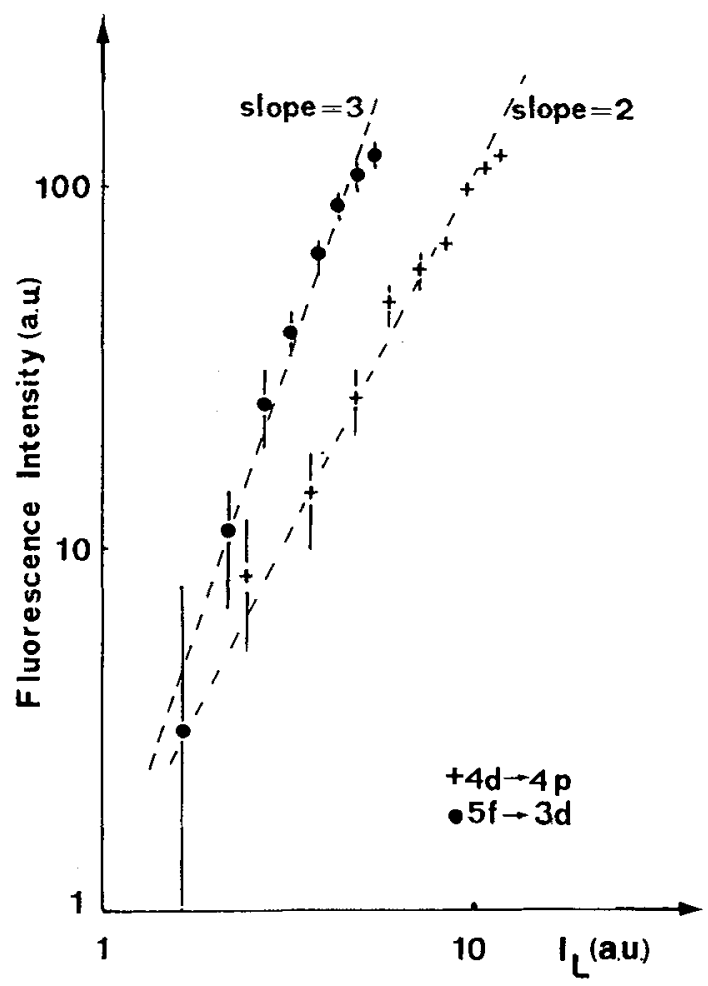

Fig. 3 - Log-log plot of the fluorescence intensity for the transitions $4 d-4 p$ and $5 f-3 d$ versus laser power. The intensities are normalized independently and the maximum laser power is $60 \mathrm{~mW}$. It is evident the square dependence of the $4 \mathrm{~d}-4 \mathrm{p}$ transition and the cubic dependence of the $5 f-3 d$ transition. 
IV - CONCLUSIONS

Since the first observations of energy pooling processes in laser-irradiated alkali vapors a great deal of work by many research groups has yield a clear insight of the phenomenon and reliable quantitative results. For convenience we report in the following table the values for energy transfer and associative ionization cross sections in the alkalis, updated to include the most recent results we are aware of:

TABLE 1. ENERGY POOLING CROSS SECTIONS OF ALKALI METAL ATOMS

A. Energy Transfer

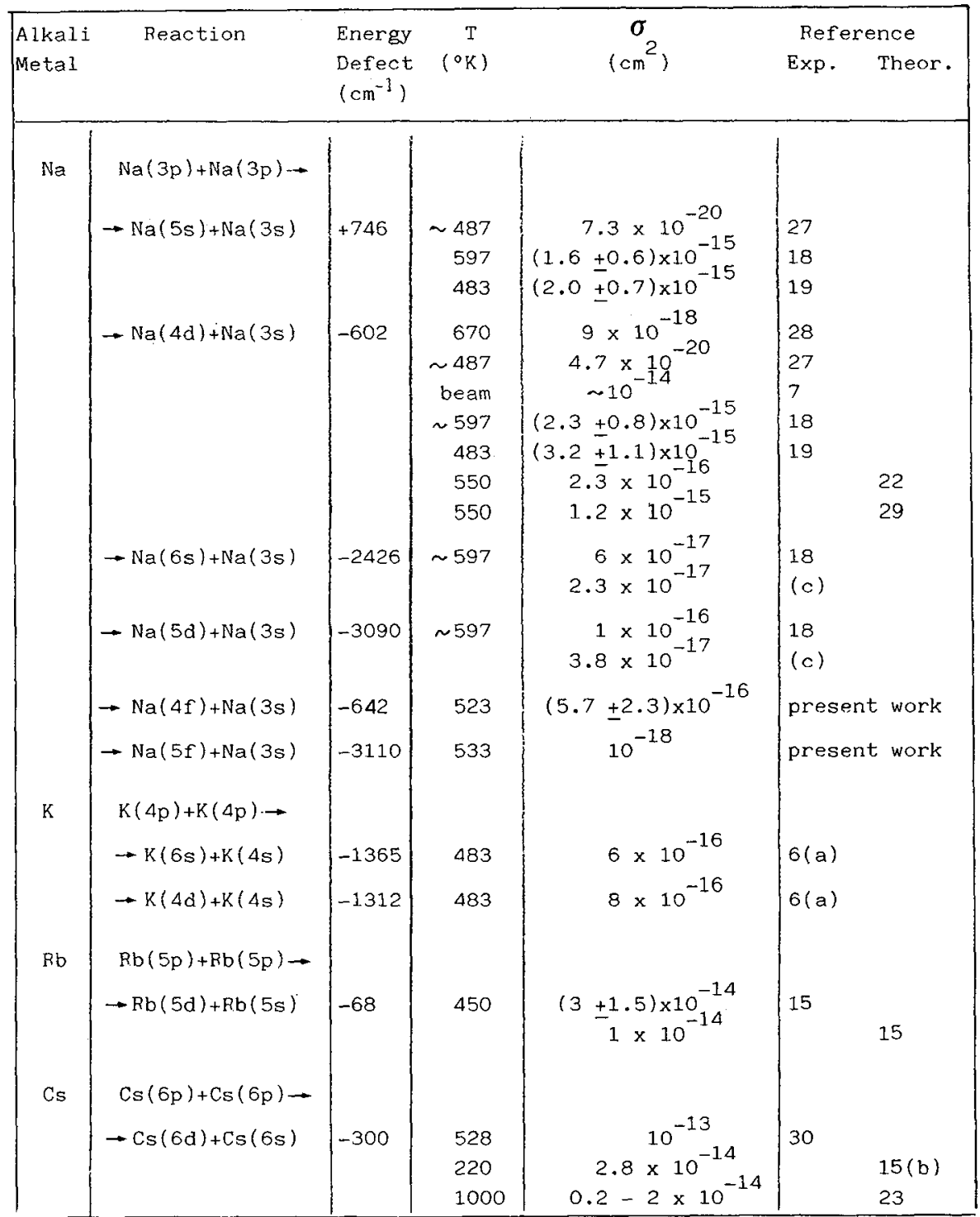


B. Associative Ionization

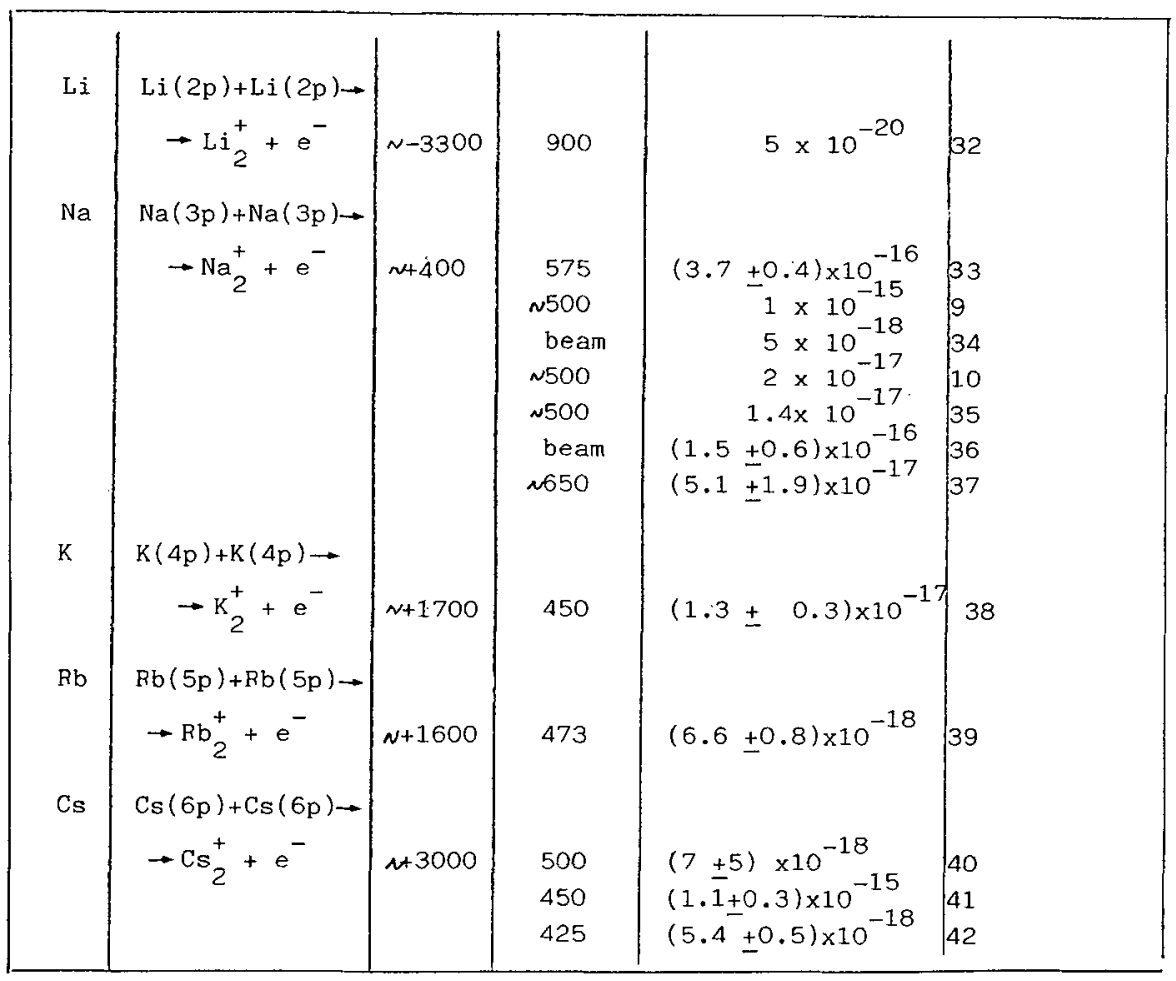

a) Deduced from the fluorescence intensity ratios $I(6 s-4 p) / I(4 p-4 s)$ and $I(4 d-4 p) / I(4 p-4 s)$ measured in ref./4/.

b) Deduced from the result of ref./31/ for the process $\operatorname{Cs}\left(6 d_{3 / 2}\right)+\operatorname{Cs}_{6 s}(1 / 2) \rightarrow$ $\operatorname{Cs}(6 p)+\operatorname{Cs}(6 p)$

c) Obtained from the absolute value of $\sigma_{5 \mathrm{~s}}$ of ref./18/ and the relative intensities given in ref./4/ at $\mathrm{T}=593^{\circ} \mathrm{K}$.

\section{REFERENCES}

1. See for example: "Photon-Assisted Collisions and Related Topics" Eds. N.K.Rahman and C.Guidotti, Harwood Academic Publishers,1982; "Collisions and Half-Collisions with Lasers" Eds. N.K.Rahman and C.Guidotti, Harwood Academic Publishers, 1984

2. Allegrini M., Garver W.P., Kushawaha V.S. and Leventhal. J.J., Phys.Rev. A28 (1983) 199 
3. Lucatorto T.B. and McIlrath T.J., Appl.Opt. 19 (1980) 3948; Kopystyńska A. and Moi L., Phys.Rep. 92 (1982) 135

4. Allegrini M., Alzetta G., Kopystynska A., Moi L. and Orriols G., Opt.Comm. 19 (1976) 96

5. Allegrini M., Gozzini S., Longo I., Savino P. and Bicchi P., Nuovo Cimento D1 (1982) 49

6. Giammanco F. and Gozzini S., Nuovo Cimento B66 (1981) 47

7. Le Gouët J.L., Picqué J.L., Wuilleumier F., Bizeau J.M., Dhez P., Koch P. and Ederer D.I., Phys.Rev.Lett. 48 (1982) 600

8. Lucatorto T.B. and McIlrath T.J., Phys.Rev.Lett. 37 (1976) 428

9. Bearman G.H. and Leventhal J.J., Phys.Rev.Lett. 41 (1978) 1227

10. Kushawaha V.S. and Leventhal J.J., Phys.Rev. A22 (1980) 2468

11. Carré B., Roussel F., Breger P. and Spiess G., J.Phys.B: At.Mol.Phys. 14 (1981) 4271; 14 (1981) 4289

12. Weiner J. and Polak-Dingels P., J.Chem.Phys. 74 (1981) 508

13. Allegrini M. and Moi L., Opt.Comm. 32 (1980) 91

14. Chéret M., Barbier L., Lindinger W. and Deloche R., J.Phys.B: At.Mol.Phys. 15 (1982) 3463

15. Barbier L. and Chéret M., J.Phys.B: At.Mo1.Phys. 16 (1983) 3213

16: Bonanno R., Boulmer J and Weiner J., Phys.Rev. A28 (1983) 604

17. Garver W.P., Pierce M.R. and Leventhal J.J., J.Chem.Phys. 77 (1982) 1201

18. Huennekens J. and Gallagher A., Phys.Rev. A27 (1983) 771

19. Allegrini M., Bicchi P. and Moi L., Phys.Rev. A28 (1983) 1338

20. Holstein T., Phys.Rev. 72 (1947) 1212; 83 (1951) 1159; Van Trigt C. Phys.Rev. 181 (1969) 97

21. Milne E.A., J.London Math.Soc. 1 (1926) 40

22. Kowalczyk P., Chem. Phys.Lett. 68 (1979) 203; 74 (1980) 80

23. Borodin V. and Komarov I.V., Opt.Spectrosc. 36 (1974) 145

24. Colle R. and Salvetti 0 ., private communication

25. Hasted J.B., Physics of Atomic Collisions, London Butterworths, 1972

26. Krasinski J., Stacewicz T. and Stroud C.R., Opt.Comm. 33 (1980) 158

27. Kushawaha V.S. and Leventhal. J.J., Phys.Rev. A25 (1982) 570

28. Krebs D.J. and Schearer L.D., J.Chem.Phys. 75 (1981) 3340

29. Kowalczyk P., J.Phys.B: At.Mol.Phys. 17 (1984)

30. Klucarev A.N. and Lucatorto A.V., Opt.Spectrosc. 32 (1972) 576

31. Yabuzaki T., Tam A.C., Hou M., Happer W. and Curry S.M., Opt.Comm. 24 (1978) 305

32. Hellfeld A.V., Caddick J. and Weiner J., Phys.Rev.Lett. 40 (1978) 1369

33. Klucharev A., Sepman V. and Vuinovich V., Opt.Spectrosc. 42 (1977) 336

34. De Jong A. and Van der Valk F., J.Phys.B: At.Mol.Phys. 12 (1979) L561

35. Kushawaha V.S. and Leventhal J.J., Phys.Rev. A25 (1982) 346

36. Bonanno R., Boulmer J. and Weiner J., Phys.Rev. A28 (1983) 604

37. Huennekens J. and Gallagher A., Phys.Rev. A28 (1983) 1276

38. Klukarev A., Sepman V. and Vuinovich V., J.Phys.B: At.Mol.Phys. 10 (1977) 715

39. Borodin V.M., Klyucharev A.N. and Sepman V.Yu, Opt.Spectrosc. 39 (1975) 231

40. Alfredo G. and Kniazzch A., Bul1.Am.Phys.Soc. 11 (1966) 634

41. Kl.yucharev A.N. and Ryazanov N.S., Opt.Spectrosc. 33 (1972) 230

42. Dobrolezh B.V., Klyucharev A.N. and Sepman V.Yu, Opt.Spectrosc. 38 (1975) 630 